\title{
Women's Sense of Belonging in Computer Science Education: The Need for a Collective Response
}

\author{
Kelly Widdicks, Alice Ashcroft, Emily Winter, and Lynne Blair \\ School of Computing and Communications, Lancaster University \\ Lancaster, UK \\ \{k.v.widdicks, alice.ashcroft, e.winter, l.blair\}@lancaster.ac.uk
}

\begin{abstract}
The gender imbalance in Computing education continues to be concerning, with women hugely underrepresented in the field. Prior research on improving gender equality in the discipline discusses the need to improve women's sense of belonging. However, this has seldom included women's understanding of what a sense of belonging is for them-nor have solutions for fostering belonging been co-created with women. In this paper, we report on the findings of four innovative focus groups with thirteen women, students and staff, in a university Computer Science department-uncovering their experiences of belonging and their proposed solutions to improving it. Through these focus groups, we present the experiences impacting our participants' sense of belonging, alongside our participants' solutions for fostering belonging within Computing. From this, we discuss the need for a collective response to fostering a sense of belonging in Computer Science, specifically through having a collective understanding of the barriers to a sense of belonging, applying a collective of solutions to foster belonging, and taking collective responsibility for improving equality.
\end{abstract}

\section{CCS CONCEPTS}

- Social and professional topics $\rightarrow$ Women; Computing education.

\section{KEYWORDS}

belonging, sense of belonging, ED\&I, gender equality, women in Computer Science education, co-creation

ACM Reference Format:

Kelly Widdicks, Alice Ashcroft, Emily Winter, and Lynne Blair. 2021. Women's Sense of Belonging in Computer Science Education: The Need for a Collective Response. In United Kingdom and Ireland Computing Education Research conference. (UKICER '21), September 2-3, 2021, Glasgow, United Kingdom. ACM, New York, NY, USA, 7 pages. https://doi.org/10.1145/3481282. 3481288

\section{INTRODUCTION}

The gender imbalance in UK Computer Science (CS) education continues to be concerning, with women making up only $17 \%$ of CS undergraduate students in 2019 [8] and being also underrepresented

Permission to make digital or hard copies of part or all of this work for personal or classroom use is granted without fee provided that copies are not made or distributed for profit or commercial advantage and that copies bear this notice and the full citation on the first page. Copyrights for third-party components of this work must be honored.

For all other uses, contact the owner/author(s).

UKICER '21, September 2-3, 2021, Glasgow, United Kingdom

(C) 2021 Copyright held by the owner/author(s)

ACM ISBN 978-1-4503-8568-8/21/09.

https://doi.org/10.1145/3481282.3481288 in ICT apprenticeships in the UK [37]. This failure to engage women in CS education and training is no doubt feeding into careers in the technology sector, with women comprising only $16 \%$ of IT professional occupations in the UK [36].

To address gender inequality in CS, there have been a number of initiatives, including: events or communities that celebrate women in CS (e.g. BCSWomen Lovelace Colloquium, Grace Hopper Celebration, the Association of Computing Machinery (ACM) ACM Women in Computing), the involvement of institutions and departments in gender equality commitments (e.g. the Athena Swan Charter), and the sharing of best practice for inclusive CS education such as EngageCSEdu by the National Center for Women \& Information Technology [4]. Taking these approaches further, and to advance positive change, there have been calls for the need to foster a sense of belonging in educative practices by Advance HE, and academic literature has been exploring belonging in the context of CS $[2,6,11,14,17,18,20,27]$. This prior research, however, seldom involves women in the process of understanding what a sense of belonging is and co-creating solutions to foster belonging in CS.

In this paper, we report on the findings from four innovative focus groups we conducted with thirteen women (staff and students) in CS education at a university level. These focus group were driven by the following research questions: RQ1- What are women's experiences of a sense of belonging (or not belonging)? RQ2- What solutions do women identify for increasing a sense of belonging in CS education? Our findings outline the experiences impacting our participants' sense of belonging as well as women's proposed solutions to fostering their belonging in CS education and careers-many of which are consistent with prior literature. From this, we discuss the need for a collective response to fostering women's and underrepresented groups' belonging in CS; namely through collective understanding of the barriers to a sense of belonging, the implementation of a collective of solutions to foster belonging, and collective responsibility across the CS sector.

\section{RELATED WORK}

To understand and address the gender imbalance in CS, prior work has studied how different demographic factors (particularly gender) affect how people approach CS education and careers. As an underrepresented group, women often have lower confidence than men in asking questions and participating in CS [1]. Women also rate their self-efficacy lower than men [23] and earlier during a course [13]-suggesting early failures may discourage women in the field. Suggestions for gender equality strategies include for educators in the discipline to be more aware of gender differences and varying support required [25] and for . learning environments 
to be supportive [1], and contain inclusive, unbiased educational materials [15].

Affirming a sense of belonging for women in CS has been found to be paramount to women's persistence [12] and self-efficacy [17] in the discipline. In other STEM subjects, such as Mathematics, women's sense of belonging affects both their desire to pursue the subject and their success [7]. Research has thus looked to understand women's belonging in CS and the factors which influence it. Sax et al. [27] found women had a lower sense of belonging than men for a CS course which worsened during the programme, while Mooney and Becker [18] found women's lower belonging is associated with identifying in another underrepresented group (e.g. LGBTQIA+).

Cheryan et al. [2] explored the effect of stereotypical environments on women's and men's ambient belonging and interest in CS, finding that stereotypical environments compromised women's ambient belonging and non-stereotypical environments were preferred by women and men. Similarly, Master et al. [14] found that stereotypical environments create a lower sense of belonging for women and discourage them from taking a CS course, while MetaxaKakavouli et al. [16] found that web design bias with CS stereotypes affects women's sense of belonging, yet gender-neutral designs are inclusive of women and men.

More recently, Nguyen and Lewis et al. [20] find that competitive enrollment procedures negatively impact a sense of belonging and self-efficacy of CS for degree students without prior CS experience, as well as being perceived as unwelcoming by students with prior experience. These authors therefore suggest departments should minimise enrollment pressures on students to alleviate these negative impacts [20]-a particularly important solution for women, who often choose to study CS without having prior experience in the subject [30] and may not see prior experience as necessary [35].

Other opportunities suggested by prior work to increase a sense of belonging for women and underrepresented groups in CS or STEM include positive mentor support through formal research experience [32] and showcasing Computing's communal affordances [11]. For the latter, Lewis et al. [11] note how critical this showcasing is given women and underrepresented groups usually have more desire to do social good but do not recognise CS as a way to enact those goals. Women's interest in doing social good, or social activism, has also been found by Sax et al. [28] to be a reason for the gender gap in CS. While technology advancement is improving children's interest in the field, there are still social constraints present that advantage men e.g. father-son relationships [3]. This is concerning given social encouragement has been shown to be important to women's drive in CS [21].

Qualitative research has been conducted to analyse the identity and histories of women within CS academia [22] and remove barriers to their belonging, such as overcoming stereotypes [6]. In this paper, we look to build on such work by explicitly involving women in the process of defining belonging, and co-creating solutions to foster belonging. We also focus on: recruiting women within CS as participants (rather than those who choose to leave or not enter the field) given the call for this in prior work [34]; and exploring belonging experiences beyond, as well as within, CS given that underrepresented groups' sense of belonging in CS is limited [11, 12, 20, 27].

\section{METHOD AND PARTICIPANTS}

We conducted four two-hour focus groups in January 2021 with thirteen participants split between four groups. The focus groups utilised innovation and ideation methods-building upon co-creation approaches in Human-Computer Interaction [e.g. 26], and utilising Adobe's KickBox practice [10] and Silverstein et al.'s concept trees and imaginary brainstorming [29]. The combination of these methods enabled a focus group that allowed for the ideation of problems using the structure of Kickbox Foundation [10] while also being solution focused [26]. This allowed the participants' understanding and experiences of belonging to be drawn upon in solutions for fostering belonging, creating a collective understanding with women. Group 1 (G1) included participants P1-5, G2 P6-7, G3 P8-11, and G4 P12-13. Groups were allocated dependent on participants' availability, and included 6 staff and 7 students (both undergraduate and postgraduate). Participants had to identify as women and work within the field of CS at our institution as a staff member, or undergraduate or postgraduate student. For concerns of anonymity, we do not provide any further demographic data.

Aligning with our co-creation approach, three authors of this paper were involved as participants. There is already a lack of women in CS, and omitting ourselves from recruitment would have minimised our participant pool further. Prior to the focus group, only two author-participants had prior knowledge of CS education literature, and discussions in the focus group focused on their experiences rather than their knowledge of this work; they also did not lead the analysis or writing. Given this, alongside concerns of anonymity, we have chosen not to differentiate ourselves from other participants in this paper. The author-participants were happy to waive their anonymity as named authors, but can not be identified with a particular participant number. Participants not involved in the research were incentivised by $£ 10$ vouchers and recruited via mailing lists, snowballing methods and Microsoft Teams groups (e.g. a departmental women's group). The study was ethically approved and all participants gave informed consent.

A facilitator, sketchnote taker, ${ }^{1}$ and note taker were present at each of the focus groups, in addition to the participants, which followed the schedule below. ${ }^{2}$

(1) Introductions. Participants introduced themselves, and described their background in Computing.

(2) Sense of belonging sentences. Participants wrote down what a sense of belonging meant to them in 1 minute. These were shared with the group and summarised by the facilitator.

(3) Sharing experiences: not belonging. Participants noted down as many experiences in 10 minutes where they have felt that they did not belong. These were then shared to the group.

(4) Sharing experiences: belonging. Participants noted down as many experiences in 10 minutes where they have felt that they did belong. These were then shared to the group.

(5) Categorising experiences. As a group, participants thematically categorised their identified experiences from the previous exercises of where they felt they did, and did not, belong.

\footnotetext{
${ }^{1}$ Sketchnoting [33] is a visual and engaging way of presenting notes.

${ }^{2} \mathrm{G} 1$ was conducted in two separate hour-long sessions. Reflecting on G1, we added 'Sharing experiences: belonging' to the schedule to ensure participants in groups G2-G4 could build on their positive belonging experiences for the solutions.
} 
(6) Identifying solutions for improving a sense of belonging. For the categories, participants individually noted down, and then discussed, solutions for improving a sense of belonging

(7) Applying the solutions to CS. Participants discussed how their solutions could specifically be applied to CS education.

(8) Identifying the group's priority solutions. Groups selected their top 3 solutions, discussing why these should be prioritised. ${ }^{3}$

All focus groups were conducted via Microsoft Teams and audiorecorded, then fully transcribed. Transcriptions were manually coded into initial themes by one of the researchers; these were then discussed with two other researchers and re-coded until a consensus was made for the final themes presented in this paper.

\section{FINDINGS}

In this section, we detail the experiences impacting our participants' belonging (section 4.1), and summarise their solutions for fostering women's belonging in CS (section 4.2).

\subsection{Experiences impacting a sense of belonging}

In the 'sharing experiences' sessions of the focus groups, the participants described memories and feelings of where they felt they did, and did not, belong.

4.1.1 Experiences of not belonging. We found four overarching themes negatively impacting our participants' sense of belonging.

Being different, being 'you'. Participants discussed having differences to others, and having these explicitly singled out by others or perceiving others as being judgemental. These differences included: physical differences (e.g. wearing glasses, body shapes), differing personality traits (e.g. being quieter than others), varying values or opinions (e.g. to materialism, religion), cultural differences (e.g. language barriers), and having contrasting circumstances to others (e.g. living elsewhere, having to work while studying).

Existence of expectations and norms. Participants expressed the burden of expectations from others, or norms at a societal level, that make them feel out of place. For example, P8 discussed others' expectations for her to continue specific hobbies or her high performance academic record-indicating expectations at a personal level. At a societal level, participants noted stereotypes and being questioned by others for not conforming to norms. Examples included being different to a stereotypical Computer Scientist (P4), and the assumed skills of women (P8-9) or their life goals (P11).

Being in an underrepresented group. Participants discussed how a specific characteristic (e.g. age, gender) that is underrepresented within a space (e.g. a fitness class, a CS course) can lead to feelings of self-consciousness (P2), alienation (P4-5), depression (P4) and loneliness (P5). Alongside these feelings, participants noted experiences where they were treated differently to the majority. For example, being subject to sexism as a student, at work or as a tutor (P6, P9), including others being shocked about the participants having knowledge about technology (P9, P11).

Feeling ignored or excluded. Participants described scenarios where they were ignored, unacknowledged or excluded in a group. At work, experiences included having contributions overlooked by

\footnotetext{
${ }^{3}$ This exercise was done before the previous in G2. We changed the order for G1, G3 and G4 to provide more time for participants to discuss solutions.
}

others (P2, P7, P9) or having someone else take credit for their hard work and ideas (P7, P9). For social situations, P12-13 noted feeling excluded in group settings where they did not know anyone, and P8-9 and P12-13 all reflected on the importance of first impressions and an initial sense of belonging to an environment-referring to feelings of exclusion from "a bad welcome" (P13).

4.1.2 Experiences of belonging. We found four overarching themes positively impacting our participants' sense of belonging.

Sharing commonalities with others. Participants discussed the importance of having similar interests, values, identities or other commonalities with people in a given group or setting. Examples of this included sharing a common goal, such as collectively talking about or working on an issue (e.g. equality, sustainability) they felt strongly about (P6, P9, P13). Participants also drew upon spending time with specific people, particularly friends, as they feel like they can 'connect' (P6) or 'be relaxed' (P7), with those people and 'never have to explain yourself' (P13) due to knowing so much about each other and their histories.

Feeling included and acknowledged. Participants associated a sense of belonging with being included or acknowledged for their contributions and skills. Examples included people asking the participants for advice (P7, P9), people agreeing with their thoughts and ideas (P7), and their opinions or views being taken into consideration (P8, P12). Participants emphasised the importance of: being heard and not judged (P11), listening to everyone's suggestions in a group project even if those solutions are not deemed successful (P8), and being loved or accepted despite being different (P6).

Being in a supportive environment. Participants spoke about being in a supportive space, where people encourage one another. Examples included: P9 having a support bubble during the Covid19 pandemic, P12's university tutor being attentive to students' questions and needs, and P11 being encouraged by close family and friends to pursue a master's degree and celebrated as a woman speaker at a conference by its organisers. Developing close relationships with others in an environment can make people feel more comfortable (P10), and an ambition to support others can be driven by past negative experiences of belonging (P13).

Finding where you thrive. Participants' belonging was linked to where their skills are best placed and the development of those skills. Examples included being offered the opportunity to perform in competitions (P8), feeling like they are studying the correct course (P13), receiving awards for best student performance (P9), and no longer having feelings of 'imposter syndrome' (P6-9, P13). This was described by some participants as a form of journey to a place of belonging (P9-10, P13), with P10 noting how her confidence grew as she found a technical role matching her expertise.

\subsection{Fostering a sense of belonging}

Following the 'sharing experiences' discussions, the participants shared solutions for improving women and girls' sense of belonging in CS education. We categorise these as educational (section 4.2.1) and cultural (section 4.2.2). These solutions are consistent with prior literature and we discuss this further in section 5.2. 
4.2.1 Educational solutions to women's belonging in CS. The participants suggested several education-based solutions for enhancing women's belonging in CS.

Capturing women's interests in CS. The participants emphasised the need to capture and maintain women's interest in CS (P2, P4, P7). Mechanisms for this included offering multiple topic options for projects (P2, P13) that form commonalities between the student and the subject. By offering multiple choices, students can follow a topic they are most interested in and avoid topics that may require an increased cognitive load (P4) or level of skill from a different discipline e.g. Maths (P1). Moreover, our participants suggested linking CS projects at school to real-world or creative problems (P2-7)-emphasising the applicability and breadth of CS (P6-7), such as through project-based teaching methods (P4-5) or by ensuring projects lead to the creation of 'tangible' and 'visual' outputs as a measure of success (P2).

Providing role models and mentors. The participants discussed the importance of role models and mentors in women's belonging in CS education (P1, P8-9, P11, P13), reflecting on how they themselves are acting as role models or sharing stories of their own mentors. For example, P8 and P9 wondered whether their parents' engagement in CS influenced them to study the field; and P9 and P11 discussed the importance of 'living by example' to inspire others, just as they have been inspired-creating a form of domino effect to widening participation. For mentorship schemes, P12 suggested meeting course alumni and P13 noted mentors may need only be $1-2$ years ahead in their career progression, as long as they are fully engaged and care about the mentee-noting a bad mentor can be worse than not having one.

Adapting teaching approaches. A supportive classroom environment that enables people to learn and develop confidence in CS was deemed important by the participants. For this, they discussed adapting teaching approaches (P5) to ensure a focus on learning outcomes (P2), and that students meet those outcomes by tutors checking in with them, fully addressing questions and creating trust in constructive criticism (P12-13). To guarantee people feel comfortable asking questions, questions should be praised with various ways to ask them (P8-9, P11, P13), such as out loud, or through anonymous submissions. Moreover, the participants suggested celebrating failure in CS (P2-5, P10)-moving the focus of being perfect first time towards 'learning by doing'. To celebrate failure: P5 suggested sharing stories of where scientists have gone wrong before their successes; and $\mathrm{P} 4-5$ discussed reframing mistakes as 'cool' or removing their individualisation, e.g. by focusing on coding 'bugs' rather than student 'mistakes'.

Adapting language. The focus group G1 participants discussed the importance of adapting language for an inclusive environmentmostly from the teachers' perspective. This solution was two-fold: 1) use gender neutral language; and 2) be aware of hidden bias in vocabulary. For the former, P1 and P3 noted how they avoid using pronouns, with $\mathrm{P} 3$ being open about her mistaken use of pronouns. For the latter, P2 highlighted how particular words are not always obviously biased-e.g. 'leadership'-but that once you consider the connotations of such words, the bias begins to become apparent. To make such bias in vocabulary more widely known, P2 suggested that there is a need for open conversations to share, discuss and learn; and P1 called for pronouns and vocabulary to be included in unconscious bias training, discussed next.

Training and development specific to CS. The participants heavily focused on the need for additional training and development for both staff and students. Participants discussed the need to not judge others (P10-11) and learn about unconscious bias (P1-3). Yet, alongside this training on current unconscious bias and inclusivity, G1 proposed education on both the history of CS and bias in CS (e.g. the history of women in CS, examples of discriminatory technology design). Adding to these topic-focused training activities, the participants advised training on mediating or setting groups in CS education settings, to ensure everyone is supported and feels comfortable (P2-5). For this, P12 discussed the importance of groups getting to know each other before they learn together, and P13 discussed making groups based on commonalities beyond diversities (e.g. favourite colour).

4.2.2 Cultural solutions to women's belonging in CS. Four overarching cultural solutions to belonging were discussed by the participants. Whilst these do overlap with specific educational solutions, these cultural solutions are also linked to broader cultural shifts required in CS to foster belong.

Building confidence: in yourself and others. Our participants discussed building people's confidence in their achievements, interests and self identify and sharing that individuality with others (P1-2, P7-11). For example, P6 noted that if people were more open to being themselves, more diverse communities would emerge: allowing for people to find people like them and feel less out of place. Through this, some participants thought that people (particularly underrepresented groups) can become more resilient to self doubt (P9) or help challenge expectations and norms by setting an example for others or acting as a role model (P1-2, P6, P8, P10-11). P11 already sets an example for others, drawing on her experience to encourage other women in her community to study.

Raising awareness of belonging barriers. In contrast to this focus on individual responsibility, some participants noted that it is important to know that you are not necessarily the issue, or a barrier, when it comes to feeling a sense of belonging. Rather, they suggested raising awareness about barriers to belonging e.g. from systemic issues in society, and emphasising that there is only so much an individual can control or change. Given this, some participants suggested leaving a particular environment or job, or trying new things to find where they belong (P7-8, P10-11); others suggested a course for action should be made beyond the individual for widening participation (P2, P5, P9) e.g. by bringing underrepresented people together, or by having 'friendly allies' in majority groups to help improve everyone's sense of belonging.

Create inclusive environments. To create a sense of belonging for everyone, participants discussed the idea of creating an inclusive and supportive environment-ensuring everyone's needs are being met (P5, P12), that people are treating each other with respect regardless of differences (P5-6), and enabling open and inclusive conversations (P2-3, P8-10). Mechanisms for this included: noticing when others feel uncomfortable and reaching out to them (P5, P8); and establishing ground rules for interactions (P6), such as ensuring everyone has time to speak, be listened to, or get to know one another (P2, P6-7, P13). P12 and P13 noted the importance of 
a 'good welcome' to events or groups, suggesting there should be specific roles or initiatives dedicated to making you feel included in a space as you arrive (e.g. a buddy scheme).

Engagement in equality, diversity and inclusion. To enact belonging solutions, the participants discussed how everyone should have access to specific training and development opportunities surrounding equality, diversity and inclusion (ED\&I). These included engaging people within a community to take unconscious bias training (P2, P4, P6), or join mentorship initiatives that can help you or others (P9). There was also discussion of people taking responsibility for learning about diversity. P4 highlighted that this is important for recognising our own limitations in ED\&I and beginning to address those- a potential first step to the participants solutions of challenging stereotypes, embracing diversity, and not judging ourselves or others.

\section{DISCUSSION AND IMPLICATIONS}

From our findings, there are three important implications for fostering a sense of belonging in CS education, specifically for women: 1) having a collective understanding of the barriers to a sense of belonging; 2) applying a collective of solutions to foster belonging; and 3) taking collective responsibility for improving equality.

\subsection{Collective understanding}

Through the focus groups, we were able to uncover an overview of the experiences impacting our participants' sense of belonging (section 4.1). We believe it is important to establish this understanding of what belonging actually 'is' to underrepresented groups as a spectrum of experiences and impacts, gathered directly with these groups-rather than relying on definitions, interpretations, and general understandings in the literature or interweaving concepts such as confidence or ability in CS [cf. 17, 18, 20,27]. This is because a sense of belonging is multi-faceted and represents individuals' lived experiences. With an in-depth consideration of belonging, we can work towards effectively fostering felt experiences of belonging and evaluating solutions against them.

Moving forward, we encourage researchers and educators to build upon our participants' experiences and consider a range of experiences of belonging in CS across all diversities. For this, we suggest the community take a two-fold consideration for a collective understanding of the issues and opportunities for belonging. Firstly, we note the importance of collating a sense of belonging beyond CS. In our focus groups, we asked participants to draw upon experiences where they felt they did or did not belong at any point in their livesbeing explicit about the fact these could be experiences beyond CS With that came a variety of experiences, drawing attention to the complexities of a sense of belonging. Taking a CS-specific focus alone would not have illuminated all the experiences we present in our paper, if not only because women already have a low sense of belonging in CS and other science disciplines [11, 12, 20, 27] and thus positive experiences of belonging in CS could arguably be limited. A collective understanding of belonging across people's lives allows for a more comprehensive picture of what exactly the CS sector needs to aim towards-bringing together solutions suggested across the literature (see section 5.2).
Secondly, we suggest it is paramount to involve underrepresented groups in diversity in CS research. The benefits of qualitative research are not unknown in this domain $[3,6,9,35]$, yet we believe involving participants through collaborative methods can offer additional gains. The focus group itself seemed to create a sense of belonging for the participants: stories were shared, commonalities were found, and there was increased visibility of women in CS. Moreover, our approach can be adopted for any department, faculty or institution - across cultures or demographics - to find out their own cohorts' experiences of belonging and solutions to barriers to a sense of belonging.. By developing this collective understanding of belonging with underrepresented groups, we can better understand: what a sense of belonging is to these groups, how to foster it, and initiate belonging through the research.

\subsection{Collective solutions}

While our participants discussed a variety of solutions to fostering belonging, we recognise that these are consistent with solutions noted in prior literature. For example, to capture women's interests in CS, prior work has noted the importance of a curriculum focused on creative or real-world problems [5, 11, 27, 28] or highlighting CS's scope, career prospects or links to other disciplines $[6,21,25,34,35]$. Creating inclusive and supportive environments $[5,12,27,30]$ has also been a prominent solution, such as by increasing social engagement with peers [18], creating welcoming first impressions [20] and adapting teaching approaches, e.g. by providing different ways for asking questions [1].

Moreover, prior work has stressed: the importance of role models, mentors and encouragement [1, 6, 21, 25, 30, 32, 35]; the need to be aware of, and improve, women's low confidence or self-efficacy, and eradicate feelings of imposter syndrome (i.e. 'building confidence') $[5,6,23,25]$; and the changes required for educational materials on CS's bias [15] and histories [34], and thus the required training and development on this. Whilst not explicit in celebrating failure, Lishinski et al. [13] found early failures discourage women in CS and Smith et al. [31] discuss the need to celebrate how much hard work goes into 'good science'.

As a community aiming to widen participation in CS, we should feel positive that we do not have a lack of solutions. Our participants' suggested solutions are encouraging signs that efforts in this domain are on the right track for fostering belonging. However, we note two concerns related to this finding. Firstly, our focus groups have highlighted that there is not one quick fix or solution for fostering women's sense of belonging in CS-rather the contrary. The issue of gender equality in the field is complex, requiring systemic change to social values and stereotypes [22]; we need many solutions to address it appropriately for all women, some of which go beyond education and require a cultural shift in CS for women's equality. Secondly, our work highlights a sad truth that we do not necessarily need new solutions for fostering a sense of belonging, i.e. we are not 'missing' something-instead, we need to enact those solutions effectively. Women, and the literature, are well aware of the issues and opportunities for gender equality in CS. Yet there is still a significant gender gap, illuminating potential problems elsewhere (e.g. a solution-effect lag, a lack of will, poor solution implementation, bureaucratic processes). 
We thus accentuate that more effort is required to effectively implement solutions for fostering belonging in CS, and we emphasise the plural here-applying a collective of solutions and cohesively evaluating the impact of these. This is not a simple task given the concerted effort required to enact multiple solutions, alongside the difficulty of conducting research evaluations that consider many solutions simultaneously over time. Perhaps complicating matters further is the cultural shift for women's equality required in CS. However, we hope by drawing together solutions across the literature and building on our sense of belonging experiences (section 4.1) as evaluative criteria, CS researchers and educators are well equipped for pursuing gender equality in CS.

\subsection{Collective responsibility}

In our participants' solutions for fostering a sense of belonging, we observe that there are somewhat contradictory elements in their suggestions. For example, the participants discussed a notion of 'building confidence' and centralising themselves as needing to change their qualities or challenge norms and expectations for their belonging. Yet they also emphasised a need to raise awareness of belonging barriers-moving the focus beyond the individual, and towards a group or society. This highlights the internal struggle women, or other underrepresented groups, may face in CS education or careers: you're the issue, you're not the issue; do change, don't change; just belong, just leave.

There were some great examples of the participants being advocates for gender equality and inclusiveness themselves, including: P9 and P11 'living by example' to inspire other women or girls to study CS; P13's emphasis on making sure people feel welcomed; and P1-3's avoidance of gendered language. They are aware of issues to belonging and want to make a positive change. Yet, we raise a concern, as researchers and women: this compelling force to champion change can be a burden on underrepresented groups, and perhaps alludes to the pressure the participants put on themselves to be confident, challenge norms and be a role model. As one of the more experienced participants in CS, P2 described the need to repeatedly challenge stereotypes as "a constant sense of "why bother?', comparing the feeling to "a never ending uphill battle".

This raises the question of whose responsibility it is to foster a sense of belonging in CS? Our answer is everyone-regardless of gender, and other demographics. But the existent gender inequality in CS, as well as other inequalities such as race [20,24], indicates this collective effort is not realised in the sector. Even our participants who acknowledge the need for cultural change still suggested solutions emphasising their own individual responsibility.

We therefore suggest that, moving forward, efforts in widening CS participation and fostering belonging for all should firstly look to address this issue of responsibility awareness-emphasising the need for collective responsibility. This may involve additional training or development for staff and students, or even more targeted approaches (e.g. requiring contribution to equality, diversity and inclusion in promotion cases), but the researchers or educators in this community should identify concrete and successful approaches to raising collective responsibility. Positive change for fostering belonging in CS is unlikely to be achieved without burdening underrepresented groups until this responsibility is fully shared.

\section{LIMITATIONS}

Our study has a small sample size, yet is not unusual for qualitative research. There may be possible bias in the results given participants are also authors of this work. However, as discussed in (section 3) this aligns with our co-creation approach; these participants focused on their experiences (not the literature); two of the three author-participants with prior knowledge of the literature did not lead the analysis and writing; and we believe it is important to reflect on our own experiences as women in CS given the current gender imbalance. Furthermore, we note that we added 'Sharing experiences: belonging' for G2-4 and amended the schedule order for 'Identifying the group's priority solutions' in G1 and G3-4 (section 3); thus there may be small differences in the discussions but we did not note anything significant in our analysis.

Another limitation of this research is that intersectionality has not been explored. Morris and Bunjun [19] plainly state: 'Intersectionality is not easy. But it is necessary to try our best to do respectful and representative research that seeks innovative and inspiring solutions that actually work for specific populations who are themselves complex and diverse.' When conducting this research, our only requirement for participants was that they self-identified as women. We did not collect any data on ethnicity, race, sexual orientation, etc.. Whilst this is a limitation, to have collected this information would have risked our participants' anonymity. Such information should be gathered by larger studies in order to understand how intersectionality affects a sense of belonging.

\section{CONCLUSION}

Given the significant gender imbalance in CS education and the need to foster a women's sense of belonging to address this, we have explored women's experiences of belonging in this paper through four focus groups. While prior work has investigated a sense of belonging through quantitative and qualitative studies, our approach extends this by explicitly involving women in order to understand their sense of belonging and co-create solutions to improve belonging. From this, we outline the experiences impacting our participants' sense of belonging and provide their solutions both educational and cultural - to foster belonging in CS.

From our findings, we suggest that researchers and educators need to take a collective response to fostering belonging in CS. Future work should build upon our participants' experiences by exploring the factors impacting belonging for other diversities and cohorts, ensuring a collective understanding to the problem through two method approaches: 1) collating belonging experiences beyond $\mathrm{CS}$, given the low sense of belonging felt by underrepresented groups in the field; and 2) involving underrepresented groups in the process, helping to create a tailored response to belonging and enhance belonging during the research itself. We also note the importance of applying and evaluating a collective of solutions in parallel for fostering belonging, especially given the complex, varying experiences of belonging felt by underrepresented individuals. Finally, we suggest that future research should find ways of emphasising the collective responsibility required by everyone in CS to address belonging and equality-alleviating the burden on women or other underrepresented groups. 


\section{REFERENCES}

[1] Christine Alvarado, Yingjun Cao, and Mia Minnes. 2017. Gender Differences in Students' Behaviors in CS Classes throughout the CS Major. In Proceedings of the 2017 ACM SIGCSE Technical Symposium on Computer Science Education (Seattle, Washington, USA) (SIGCSE '17). ACM, New York, NY, USA, 27-32. https : //doi.org/10.1145/3017680.3017771

[2] Sapna Cheryan, Victoria C Plaut, Paul G Davies, and Claude M Steele. 2009. Ambient belonging: how stereotypical cues impact gender participation in computer science. Fournal of personality and social psychology 97, 6 (2009), 1045.

[3] Mathilde Collain and Deborah Trytten. 2019. "You Don't Have to Be a White Male That Was Learning How to Program since He Was Five:" Computer Use and Interest From Childhood to a Computing Degree. In Proceedings of the 50th ACM Technical Symposium on Computer Science Education (Minneapolis, MN USA) (SIGCSE '19). ACM, New York, NY, USA, 968-974. https://doi.org/ 10.1145/3287324.3287383

[4] Wendy M. DuBow, Beth A. Quinn, Gloria Childress Townsend, Rosario Robinson, and Valerie Barr. 2016. Efforts to Make Computer Science More Inclusive of Women. ACM Inroads 7, 4 (Nov. 2016), 74-80. https://doi .org/10.1145/ 2998500

[5] EngageCSEdu, The National Center for Women \& Information Technology (NCWIT). 2021. EngageCSEdu Engagement Practices. https://www.engagecsedu.org/engagement/grow-inclusive-student-community, accessed 01/21.

[6] Katrina Falkner, Claudia Szabo, Dee Michell, Anna Szorenyi, and Shantel Thyer 2015. Gender Gap in Academia: Perceptions of Female Computer Science Academics. In Proceedings of the 2015 ACM Conference on Innovation and Technology in Computer Science Education (Vilnius, Lithuania) (ITiCSE '15). ACM, New York, NY, USA, 111-116. https://doi.org/10.1145/2729094.2742595

[7] Catherine Good, Aneeta Rattan, and Carol S Dweck. 2012. Why do women opt out? Sense of belonging and women's representation in mathematics. Fournal of personality and social psychology 102, 4 (2012), 700.

[8] HESA. 2020. What do HE students study? Personal characteristics, 2019-20 data. https://www.hesa.ac.uk/data-and-analysis/students/wh at-study/characteristics, accessed $05 / 21$

[9] Lilly Irani. 2004. Understanding Gender and Confidence in CS Course Culture. SIGCSE Bull. 36, 1 (March 2004), 195-199. https://doi.org/10.1145/ 1028174. 971371

[10] Kickbox Foundation. 2021. Adobe Kickbox. https://www.kickbox.org/ adobe-kickbox-material, accessed 01/21.

[11] Colleen Lewis, Paul Bruno, Jonathan Raygoza, and Julia Wang. 2019. Alignment of Goals and Perceptions of Computing Predicts Students' Sense of Belonging in Computing. In Proceedings of the 2019 ACM Conference on International Computing Education Research (Toronto ON, Canada) (ICER '19). ACM, New York, NY, USA 11-19. https://doi.org/10.1145/3291279.3339426

[12] Karyn L. Lewis, Jane G. Stout, Noah D. Finkelstein, Steven J. Pollock, Akira Miyake Geoff L. Cohen, and Tiffany A. Ito. 2017. Fitting in to Move Forward: Belonging, Gender, and Persistence in the Physical Sciences, Technology, Engineering, and Mathematics (pSTEM). Psychology of Women Quarterly 41, 4 (2017), 420-436. https://doi.org/10.1177/0361684317720186

[13] Alex Lishinski, Aman Yadav, Jon Good, and Richard Enbody. 2016. Learning to Program: Gender Differences and Interactive Effects of Students' Motivation, Goals, and Self-Efficacy on Performance. In Proceedings of the 2016 ACM Conference on International Computing Education Research (Melbourne, VIC, Australia) (ICER '16). ACM, New York, NY, USA, 211-220. https: //doi.org/10.1145/2960310.2960329

[14] Allison Master, Sapna Cheryan, and Andrew N Meltzoff. 2016. Computing whether she belongs: Stereotypes undermine girls' interest and sense of belonging in computer science. Journal of educational psychology 108, 3 (2016), 424.

[15] Paola Medel and Vahab Pournaghshband. 2017. Eliminating Gender Bias in Computer Science Education Materials. In Proceedings of the 2017 ACM SIGCSE Technical Symposium on Computer Science Education (Seattle, Washington, USA) (SIGCSE '17). ACM, New York, NY, USA, 411-416. https://doi.org/10. $1145 / 3017680.3017794$

[16] Danaë Metaxa-Kakavouli, Kelly Wang, James A. Landay, and Jeff Hancock. 2018 Gender-Inclusive Design: Sense of Belonging and Bias in Web Interfaces. ACM, New York, NY, USA, 1-6. https://doi.org/10.1145/3173574.3174188

[17] Allison Mishkin. 2019. Applying Self-Determination Theory towards Motivating Young Women in Computer Science. In Proceedings of the 50th ACM Technical Symposium on Computer Science Education (Minneapolis, MN, USA) (SIGCSE '19). ACM, New York, NY, USA, 1025-1031. https://doi.org/10.1145/ 3287324.3287389

[18] Catherine Mooney and Brett A. Becker. 2020. Sense of Belonging: The Intersectionality of Self-Identified Minority Status and Gender in Undergraduate Computer Science Students. In United Kingdom \& Ireland Computing Education Research Conference. (Glasgow, United Kingdom) (UKICER '20). ACM, New York, NY, USA, 24-30. https://doi.org/10.1145/3416465.3416476
[19] Marika Morris and Bénita Bunjun. 2007. Using intersectional feminist frameworks in research. Ottawa, ON: Canadian Research Institute for the Advancement of Women (2007), 1-9.

[20] An Nguyen and Colleen M. Lewis. 2020. Competitive Enrollment Policies in Computing Departments Negatively Predict First-Year Students' Sense of Belonging, Self-Efficacy, and Perception of Department. In Proceedings of the 51st ACM Technical Symposium on Computer Science Education (Portland, OR, USA) (SIGCSE '20). ACM, New York, NY, USA, 685-691. https://doi.org/10. $1145 / 3328778.3366805$

[21] Abigail Powell, Andrew Dainty, and Barbara Bagilhole. 2012. Gender stereotypes among women engineering and technology students in the UK: lessons from career choice narratives. European fournal of Engineering Education 37, 6 (2012), 541-556. https://doi.org/10.1080/03043797.2012.724052

[22] Rose K. Pozos and Michelle Friend. 2021. "You Sound Like a Good Program Manager": An Analysis of Gender in Women's Computing Life Histories. In Proceedings of the 52nd ACM Technical Symposium on Computer Science Education (Virtual Event, USA) (SIGCSE '21). ACM, New York, NY, USA, 692-698. https: //doi.org/10.1145/3408877.3432433

[23] Keith Quille, Natalie Culligan, and Susan Bergin. 2017. Insights on Gender Differences in CS1: A Multi-Institutional, Multi-Variate Study.. In Proceedings of the 2017 ACM Conference on Innovation and Technology in Computer Science Education (Bologna, Italy) (ITiCSE '17). ACM, New York, NY, USA, 263-268. https://doi.org/10.1145/3059009.3059048

[24] Yolanda A. Rankin and Jakita O. Thomas. 2020. The Intersectional Experiences of Black Women in Computing. In Proceedings of the 51st ACM Technical Symposium on Computer Science Education (Portland, OR, USA) (SIGCSE '20). ACM, New York, NY, USA, 199-205. https://doi.org/10.1145/3328778.3366873

[25] Katie Redmond, Sarah Evans, and Mehran Sahami. 2013. A Large-Scale Quantitative Study of Women in Computer Science at Stanford University. In Proceeding of the 44th ACM Technical Symposium on Computer Science Education (Denver, Colorado, USA) (SIGCSE '13). ACM, New York, NY, USA, 439-444. https://doi .org/10.1145/2445196.2445326

[26] Elizabeth B-N Sanders and Pieter Jan Stappers. 2008. Co-creation and the new landscapes of design. Co-design 4, 1 (2008), 5-18.

[27] Linda J. Sax, Jennifer M. Blaney, Kathleen J. Lehman, Sarah L. Rodriguez, Kari L. George, and Christina Zavala. 2018. Sense of Belonging in Computing: The Role of Introductory Courses for Women and Underrepresented Minority Students. Social Sciences 7, 8 (2018). https://doi.org/10.3390/socsci 7080122

[28] Linda J. Sax, Kathleen J. Lehman, Jerry A. Jacobs, M. Allison Kanny, Gloria Lim, Laura Monje-Paulson, and Hilary B. Zimmerman. 2017. Anatomy of an Enduring Gender Gap: The Evolution of Women's Participation in Computer Science. The Journal of Higher Education 88, 2 (2017), 258-293. https://doi .org/10. 1080/00221546.2016.1257306

[29] David Silverstein, Philip Samuel, and Neil Decarlo. 2013. The Innovator's Toolkit (2nd ed.). Hoboken, N.J.: Wiley. https://doi.org/10.1002/ $\mathbf{9 7 8 1 1 1 8 2 5 8 3 1 6}$

[30] Jane Sinclair and Sara Kalvala. 2015. Exploring Societal Factors Affecting the Experience and Engagement of First Year Female Computer Science Undergraduates. In Proceedings of the 15th Koli Calling Conference on Computing Education Research (Koli, Finland) (Koli Calling '15). ACM, New York, NY, USA, 107-116. https://doi.org/10.1145/2828959.2828979

[31] Jessi L. Smith, Karyn L. Lewis, Lauren Hawthorne, and Sara D. Hodges. 2013. When Trying Hard Isn't Natural: Women's Belonging With and Motivation for Male-Dominated STEM Fields As a Function of Effort Expenditure Concerns. Personality and Social Psychology Bulletin 39, 2 (2013), 131-143. https://doi . org/10.1177/0146167212468332 PMID: 23187722 .

[32] Jane G. Stout, N. Burçin Tamer, and Christine J. Alvarado. 2018. Formal Research Experiences for First Year Students: A Key to Greater Diversity in Computing? In Proceedings of the 49th ACM Technical Symposium on Computer Science Education (Baltimore, Maryland, USA) (SIGCSE '18). ACM, New York, NY, USA, 693-698. https://doi.org/10.1145/3159450.3159472

[33] Miriam Sturdee, Makayla Lewis, and Nicolai Marquardt. 2018. SketchBlog \#1: The Rise and Rise of the Sketchnote. Interactions 25, 6 (Oct. 2018), 6-8. https: //doi.org/10.1145/3281661

[34] Anna Vitores and Adriana Gil-Juárez. 2016. The trouble with 'women in computing': a critical examination of the deployment of research on the gender gap in Computer Science. Journal of Gender Studies 25, 6 (2016), 666-680. https://doi.org/10.1080/09589236.2015.1087309

[35] Sarah Theres Völkel, Wiktoria Wilkowska, and Martina Ziefle. 2018. GenderSpecific Motivation and Expectations toward Computer Science. In Proceedings of the 4th Conference on Gender \& IT (Heilbronn, Germany) (GenderIT '18). ACM, New York, NY, USA, 123-134. https://doi.org/10.1145/ 3196839.3196858

[36] WISE. 2019. 2018 Workforce Statistics. https://www. wisecampaign.org .uk/statistics/2018-workforce-statistics/, accessed 01/21.

[37] WISE. 2019. Women in STEM Apprenticeships 2017/18. https: //www.wisecampaign.org.uk/statistics/women-in-stemapprenticeships-2017-18/, accessed 01/21. 